\title{
Traditional Chinese Medicine for Refractory Nephrotic Syndrome: Strategies and Promising Treatments
}

\author{
Xiao-Qin Wang $\mathbb{D}^{1,2}$ Lan Wang $\mathbb{D}^{1,2}$ Yuan-Chao Tu, ${ }^{1,2}$ and Yuan Clare Zhang ${ }^{3}$ \\ ${ }^{1}$ Department of Chinese Medicine Nephrology, Hubei Provincial Hospital of TCM, Hubei University of Chinese Medicine, \\ Wuhan, Hubei, China \\ ${ }^{2}$ Institute of Chinese Medicine Nephrology, Hubei Province Academy of Traditional Chinese Medicine, Wuhan, Hubei, China \\ ${ }^{3}$ Y. Clare Zhang Practice of Oriental Medicine, Tucson, AZ, USA
}

Correspondence should be addressed to Xiao-Qin Wang; wangxiaoqin@hbhtcm.com and Yuan Clare Zhang; drclare@clarehealth.com

Received 27 September 2017; Accepted 3 December 2017; Published 4 January 2018

Academic Editor: Young-Su Yi

Copyright (c) 2018 Xiao-Qin Wang et al. This is an open access article distributed under the Creative Commons Attribution License, which permits unrestricted use, distribution, and reproduction in any medium, provided the original work is properly cited.

Refractory nephrotic syndrome (RNS) is an immune-related kidney disease with poor clinical outcomes. Standard treatments include corticosteroids as the initial therapy and other immunosuppressants as second-line options. A substantial proportion of patients with RNS are resistant to or dependent on immunosuppressive drugs and often experience unremitting edema and proteinuria, cycles of remission and relapse, and/or serious adverse events due to long-term immunosuppression. Traditional Chinese medicine has a long history of treating complicated kidney diseases and holds great potential for providing effective treatments for RNS. This review describes the Chinese medical theories relating to the pathogenesis of RNS and discusses the strategies and treatment options using Chinese herbal medicine. Available preclinical and clinical evidence strongly supports the integration of traditional Chinese medicine and Western medicine for improving the outcome of RNS. Herbal medicine such as Astragalus membranaceus, Stephania tetrandra S. Moore, and Tripterygium wilfordii Hook F can serve as the alternative therapy when patients fail to respond to immunosuppression or as the complementary therapy to improve therapeutic efficacy and reduce side effects of immunosuppressive agents. Wuzhi capsules (Schisandra sphenanthera extract) with tacrolimus and tetrandrine with corticosteroids are two herb-drug combinations that have shown great promise and warrant further studies.

\section{Refractory Nephrotic Syndrome-A Rare Immune Disease with Serious Consequences}

Nephrotic syndrome is a rare but serious kidney disease that affects children and adults worldwide. Its clinical presentations include peripheral edema, heavy proteinuria, and hypoalbuminemia, often with hyperlipidemia. The reported annual incidence is $2-7$ per 100,000 children and 3 per 100,000 adults [1,2]. Although occurring at a low rate, it is responsible for approximately $12 \%$ of all causes of end stage renal disease (ESRD) and up to $20 \%$ of ESRD in children [3]. The etiology of nephrotic syndrome ranges from primary glomerulonephritis to secondary diseases associated with drugs, infections, and neoplasia. The cause of primary nephrotic syndrome is complex and not well understood; however ample evidence indicates that it is an immune-mediated disorder leading to glomerular podocyte injury and increased glomerular permeability [4].

Primary nephrotic syndrome consists of three major pathophysiological subtypes-idiopathic membranous nephropathy (IMN), minimal change disease (MCD), and focal segmental glomerulosclerosis (FSGS). Their pathogenic mechanisms, albeit different in aspects, all involve immune damage of glomerular podocytes with low inflammatory nature.

IMN is generally considered an autoimmune disease, characterized by immune complex deposition and complement activation in the subepithelial space between glomerular podocytes and the glomerular basement membrane, which together contribute to functional disruption of the glomerular capillary wall [5]. The identification of two important podocyte autoantigens-secretory phospholipase 
A2 receptor (PLA2R1) and thrombospondin type 1 domain containing 7A protein (THSD7A) - and detection of their autoantibodies in $60-80 \%$ and $5-10 \%$ of patients with membranous nephropathy, respectively $[6,7]$, were regarded as landmark discoveries in understanding the molecular pathomechanism of IMN.

MCD and FSGS were traditionally described as separate entities. However, convincing evidence now suggests that they are in fact different manifestations of the same progressive disease, with FSGS being the more advanced stage than MCD [8]. Their immune pathogenesis is considered to arise from a systemic disturbance of T-cell function leading to the production of cytokines or other circulating permeability factors that cause direct or indirect impairment of glomerular function [9].

IMN is the leading cause of nephrotic syndrome in adults, but it is rare in children [1]. MCD and FSGS are the most common causes of childhood nephrotic syndrome. In adults, they each account for $10-15 \%$ and $40 \%$ of nephrotic syndrome cases, respectively [10].

The standard first-line treatment for nephrotic syndrome is corticosteroids. Although most children with nephrotic syndrome are sensitive to steroids, approximately $20 \%$ of children are steroid-resistant. Moreover, $80-90 \%$ of pediatric patients who respond initially to steroids experience relapse, and many develop steroid dependency following frequent relapses and repeated courses of steroid administration [11]. Refractory nephrotic syndrome (RNS) thus refers to the subset of patients with nephrotic syndrome who are steroidresistant, or steroid-dependent, and experience frequent relapses [12]. RNS comprises $25-40 \%$ of nephrotic syndrome cases in children and adolescents [13], and its incidence is even greater (up to 70\%) in adults [10]. For instance, MCD has over $50 \%$ relapse rate in adults [10], and $10-20 \%$ of the adult patients with MCD are steroid-resistant [14]. Due to the high incidence of RNS in adult nephrotic syndrome, the terms of nephrotic syndrome and RNS are often used interchangeably in medical practice and literature.

In recent years, second-line immunosuppressive therapies including cytotoxic agents, calcineurin inhibitors, mycophenolate mofetil, and rituximab have been widely used in RNS treatment with promising results. However, longterm use of steroids and some of these immunosuppressants causes serious adverse effects such as nephrotoxicity, hyperglycemia, dyslipidemia, osteoporosis, hypoimmunity, and high risk of infection. Moreover, the risk/benefit profile of these treatments is poor. Most patients with RNS still present severe nonremitting edema, heavy proteinuria, hypoalbuminemia, and sometimes decreased kidney function.

The limited success of currently available treatments for RNS has prompted active research into safer and more effective alternative therapies. Traditional Chinese medicine (TCM) has a long history of treating symptoms of kidney diseases such as edema and proteinuria. Through persistent trial and error, theorization, and retheorization, TCM has developed unique perspectives to explain nephrotic syndrome and accumulated rich clinical experience in treating this disease. Therefore, it makes sense to explore TCM for better RNS treatment.
In this article, we briefly review the current status of RNS treatment by conventional medicine. We describe the TCM theory for understanding the etiology and pathogenesis of RNS. We also discuss the TCM strategies and treatment options as alternative therapies or in a supportive role to improve the efficacy and reduce side effects of immunosuppressive drugs. Finally, we present two herb-drug combinations with promising clinical results and discuss possible mechanisms of action in the treatment of RNS.

\section{Current Status of RNS Treatment}

For over 40 years, empirical treatment with immunosuppressive agents including corticosteroids has been the mainstay of therapy for nephrotic syndrome [15]. This is largely due to insufficient knowledge of the detailed molecular and cellular mechanisms underlying nephrotic syndrome and thus lack of specific therapeutic targets and treatment guidelines. Steroids remain the standard initial therapy. Depending on the response to steroids, patients fall into steroid-sensitive and steroid-resistant groups. Steroid-resistant patients have a high risk of developing renal failure, while a substantial portion of steroid-sensitive patients experience frequent relapses, develop steroid dependency, and suffer a myriad of serious adverse effects from repeated steroid use. These patients are difficult to treat and face a long battle with RNS.

In order to improve clinical outcomes in patients with RNS, alternative immunosuppressive treatments have been introduced in recent decades, including cytotoxic drugs such as cyclophosphamide, lymphocyte DNA synthesis inhibitors such as mycophenolate mofetil, and calcineurin inhibitors such as cyclosporin and tacrolimus. These agents can be used alone or in combination with steroids. However, the rate of remission induced by different regimens has been highly variable in clinical trials, resulting in a lack of clear consensus regarding the comparative advantages of various combinations. Almost all of the immunosuppressive drugs produce toxic adverse effects that counter their therapeutic benefits. Furthermore, some long-term studies (follow-up for over 10 years) have failed to demonstrate greater remission rates with immunosuppressive therapy than with conservative therapy without immunosuppression [16]. Therefore, the overall risk-benefit profile of immunosuppressive therapy for RNS remains poor.

Considerable progress over the past decade in understanding the molecular mechanisms of nephrotic syndrome has enabled development and testing of novel immunotherapies [17]. Of particular importance is the use of specific Bcell targeting monoclonal antibodies such as rituximab [18]. In several studies, rituximab successfully improved remission rates in patients that had become dependent on other immunosuppressive drugs [18-21]. Based on the hypothesis of circulating permeability factors underlying FSGS and MCD, plasmapheresis has also been attempted in FSGS patients as an alternative approach for treating recurrent nephrotic syndrome following kidney transplantation [22].

Despite the promise that rituximab offers in the treatment of RNS, the available evidence of its efficacy is derived primarily from a few short-term small case studies which 
showed mixed results $[23,24]$. More research is warranted to confirm its efficacy and to observe its long-term safety profile. The prohibitive unit cost of rituximab is also an obstacle to its wide use [25]. Irrespective of different types of conventional therapies and new treatments, a majority of RNS patients still face frequent relapses. Moreover, toxic adverse effects associated with long-term use of immunosuppressive agents continue to present a serious challenge in the clinical management of RNS.

\section{TCM Views and Treatments for RNS}

Because severe edema is the most direct manifestation of RNS, the understanding of RNS in TCM has been based primarily on the theory and clinical experience in treating edema. The pathogenesis of edema was first described in Yellow Emperor's Inner Classics, a book written before 100 BC that established the theoretical foundation of TCM [26]. Edema was thought to arise from functional disturbance of three organ systems including Lungs, Spleen, and Kidneys, and the consequent disruption of fluid metabolism. In order to better understand TCM theories, it is important to point out that the definition of organs in TCM is different from that of Western medicine. Organs in the TCM context often constitute functional organ systems with both anatomical and functional meanings. Lungs represent the respiratory system that controls breath and perspiration, Spleen represents the digestive system that controls food assimilation and feces elimination, and Kidneys represent the urinary system that controls urine excretion. A myriad of factors can lead to disorders of these three systems, which separately or collectively result in disruption of fluid homeostasis leading to edema. Therefore, the fundamental TCM treatment strategy for edema is to regulate the functions of Lungs, Spleen, and Kidneys in order to promote fluid elimination through sweat, feces, and urine. When edema is not quickly resolved and becomes chronic, it can block energy flow and blood circulation and thus cause pathological patterns such as Damp Heat, Heat Toxins, Qi Stagnation, and Blood Stasis, leading to secondary complications such as localized or systemic inflammation, ischemia, swelling, pain, stiffness, and skin ulcers. When the root causes of edema and the associated complications are treated accordingly, better therapeutic effects can be achieved [27].

What determines the prognosis, remission, and relapse of nephrotic syndrome? Why are some patients more difficult to treat than others? From the TCM perspective, autoimmune diseases are largely considered to be constitutional diseases. Constitutional characteristics are the result of multiple factors, among which genetics is the decisive factor conferring relative stability and predictability to the constitution. Meanwhile, a person's constitution can be affected by other factors leading to its changeability. This concept is the basis for individuality of disease pathogenesis and responses to treatments. According to the TCM principles first described in Yellow Emperor's Inner Classics, personalized treatments must be based on duration of diseases, severity of symptoms, and body constitutions. One of the most important principles is avoiding damaging the patient's Upright Qi, which means a person's internal energy and immunity. In other words, Upright Qi determines the likelihood of remission and relapse of difficult-to-treat diseases. This notion is consistent with the knowledge of disease prognosis in modern medicine, for instance, in cancer treatment [28].

Why is RNS difficult to treat? The reason is its complexity. In the scenario of RNS, symptoms such as severe edema, heavy proteinuria, and hypoalbuminemia have not resolved; serious adverse effects of steroids and other immunosuppressants have persisted; and Upright Qi has been damaged which makes the disease resolution even more difficult. From the perspective of Yin-Yang balance, nephrotic syndrome is mainly characterized as Yang Deficiency and Yin Excess. When nephrotic syndrome becomes RNS, Yang often becomes more deficient, thus worsening the imbalance of Yin-Yang. Other pathological factors such as accumulation of toxins and Blood Stasis (i.e., poor blood circulation) further complicate treatments. Therefore, the crucial TCM concept in treating RNS is to regulate Yin-Yang and Qi-Blood, stabilize the internal environment, and avoid damaging Upright Qi by overtreatment. The comprehensive goal is to maintain biological homeostasis, improve blood circulation and eliminate toxins, minimize the doses and adverse effects of immunosuppressants, improve clinical symptoms, reduce proteinuria, and protect kidney functions in order to improve the overall survival and quality of life of patients with RNS [27].

The TCM strategy for treating edema was first established by Zhang Zhong-Jing (150-219 AD), the most eminent physician in the Chinese history [29]. For readers unfamiliar with Chinese medicine, the significance of Zhang Zhong-Jing and his work can be exemplified by a simple fact that Kampo medicine widely practiced in Japan still uses his original herb formulations with only the slightest changes. The one hundred forty-eight Kampo formulations approved by Japan's national healthcare system are mostly based on his work. These ancient formulations continue to prove valuable for treating modern diseases.

Zhang Zhong-Jing designed many herb formulas to treat edema with varying severities and symptoms (Table 1). Due to the exceptional efficacy of these formulas, they have served as the base formulas for treating edema with subsequent modifications by later generations of physicians. A review of these traditional formulas reveals that Stephania tetrandra S. Moore (Fang Ji) and Astragalus membranaceus (Huang Qi) are the most important herbs for treating edema.

The clinical effectiveness of Astragalus membranaceus in nephrotic syndrome has been consistently demonstrated in many clinical studies [30]. Astragalus is commonly used in clinical management of proteinuria of various etiologies and is especially effective in glomerulonephritis and nephrotic syndrome [31]. In one study conducted in China, 30 patients with chronic glomerulonephritis, among whom nine were diagnosed with RNS including eight with FSGS and one with IMN, received Astragalus therapy [32]. Following 3 weeks of intravenous injections of Astragalus extract at $80 \mathrm{~g} /$ day, marked reductions in proteinuria were observed in 6 of 8 patients with FSGS, but not in the one patient with IMN. In two separate case reports, patients with 
TABLE 1: Traditional herb formulas and ingredients for treating edema.

\begin{tabular}{lcc}
\hline Indications & Formulas & Herbs \\
Mild edema & $\begin{array}{c}\text { Stephania \& Astragalus } \\
\text { Decoction (Fang Ji Huang } \\
\text { Qi Tang) }\end{array}$ & $\begin{array}{c}\text { Stephania tetrandra (Fang Ji), Astragalus membranaceus (Huang Qi), } \\
\text { Atractylodes macrocephala (Bai Zhu), Glycyrrhiza uralensis (Gan Cao) }\end{array}$ \\
$\begin{array}{lcc}\text { Moderate edema } \\
\text { Severe edema }\end{array}$ & $\begin{array}{c}\text { Stephania tetrandra (Fang Ji), Astragalus membranaceus (Huang Qi), } \\
\text { (Fang Ji Fu Ling Tang) }\end{array}$ & $\begin{array}{c}\text { Glycyrrhiza uralensis (Gan Cao), Poria cocus (Fu Ling), Cinnamomum } \\
\text { cassia (Gui Zhi) }\end{array}$ \\
\hline $\begin{array}{l}\text { Cocculus decoction (Mu } \\
\text { Fang Ji Tang) }\end{array}$ & $\begin{array}{c}\text { Cocculus orbiculatus (Mu Fang Ji), Panax ginseng (Ren Shen), } \\
\text { Cinnamomum cassia (Gui Zhi), Gypsum (Shi Gao) }\end{array}$ \\
$\begin{array}{l}\text { Cocculus minus Gypsum } \\
\text { plus Poria plus Mirabilite } \\
\text { decoction (Mu Fang Ji Qu } \\
\text { Shi Gao Jia Fu Ling Mang } \\
\text { Xiao Tang) }\end{array}$ & $\begin{array}{c}\text { Stephania tetrandra (Fang Ji), Panax ginseng (Ren Shen), } \\
\text { Cinnomum cassia (Gui Zhi), Mirabilite (Mang Xiao), Poria cocos }\end{array}$ \\
$\begin{array}{l}\text { Severe edema with } \\
\text { constipation and } \\
\text { urinary retention }\end{array}$ & $\begin{array}{c}\text { Stephania Zanthoxylum } \\
\text { Descurainia Rhubarb Pill (Ji } \\
\text { Jiao Li Huang Wan) }\end{array}$ & $\begin{array}{c}\text { Stephania tetrandra (Fang Ji), Zanthoxylum bungeanum (Jiao Mu), } \\
\text { Descurainia sophia (Ting Li Zi), Rheum officinale (Da Huang) }\end{array}$ \\
\hline
\end{tabular}

RNS due to IMN who had previously failed to respond to immunosuppressive therapy and supportive care were able to achieve complete remission after taking Astragalus $[33,34]$. For example, a 77-year old patient was treated with supportive therapy (angiotensin-converting enzyme inhibitor, angiotensin receptor blocker, statin, and diuretics) and immunosuppressive agents (cyclosporin and mycophenolate mofetil) for 1 year without any response. After 2 years of unremitting nephrosis, she began oral administration of a Chinese herbal medicine Nephritis Four-ingredient Pill (Shen Yan Si Wei Pian) with the active ingredient of Astragalus at a dose of $15 \mathrm{~g} /$ day. Proteinuria, hypoalbuminemia, hypercholesterolemia, and edema all resolved after approximately 1 year of therapy, and the remission persisted for the next 4 years [33].

Likewise, Stephania tetrandra has been widely used for treating nephrotic syndrome and chronic kidney disease, usually in combination with Astragalus. In a double-blinded randomized clinical trial (RCT) involving 578 patients with glomerulonephritis in stage 3 chronic kidney disease, we treated patients for 24 weeks with benazepril, a herbal medicine Stephania, and Astragalus Decoction (Fang Ji Huang Qi Tang), or the combination of these two [35]. Results demonstrated that the herbal medicine improved renal function shown by significant increases in estimated glomerular filtration rate (eGFR) and hemoglobin with the lowest incidence of side effects; benazepril decreased proteinuria; and the benazepril-herb combination had synergistic effects on reducing proteinuria and protecting kidney functions. Many other studies evaluating Stephania and Astragalus Decoction in nephrotic syndrome have reported similar benefits in reducing proteinuria and hyperlipidemia and increasing serum albumin [36-38]. Circulating cytokine levels were affected by the herbal medicine with significant decreases in TNF- and IL-6 and increases in IL-10 [36, 37].

Shenqi particle is a Chinese herbal medicine consisting of 13 herbs including Astragalus. Since the 1980s, it has been successfully used to treat various immune-related kidney diseases. Previous small clinical studies showed that Shenqi particle and its components reduced proteinuria in patients with membranous nephropathy $[39,40]$. A prospective RCT was conducted in 190 adult patients with membranous nephropathy to compare the efficacy and safety of Shenqi particle with standard therapy of prednisone and cyclophosphamide [41]. After 48 weeks of treatment, both groups showed comparable levels of reduction in proteinuria and similar improvement in serum albumin. However, patients treated with Shenqi particle had significantly higher eGFR compared with baseline, while eGFR was slightly decreased in patients receiving standard therapy. Furthermore, severe adverse events occurred only with standard therapy. These results indicate that Shenqi particle has similar efficacy but fewer side effects compared to standard therapy. It also protects kidney function, while standard therapy does not. Therefore, Shenqi particle is a promising alternative therapy for RNS.

There are more ongoing trials of Chinese herbal medicine in RNS. A double-blinded RCT is currently evaluating the effectiveness of QingReMoShen granules in combination with angiotensin II receptor blocker in the reduction of proteinuria and T lymphocytes in IMN (clinicaltrials.gov, NCT01845688).

\section{TCM to Support Immunosuppressive Therapy for RNS}

As mentioned previously, in TCM theories the basic body constitution for nephrotic syndrome is primarily Yang Deficiency leading to fluid retention with secondary complications such as Blood Stasis. The standard therapy for nephrotic syndrome in conventional medicine is corticosteroids combined with other immunosuppressive drugs. These immunosuppressive therapies often shift the pathology of nephrotic syndrome toward further disequilibrium of Yin-Yang. In the TCM perspective, corticosteroids are pure Yang agents with hot nature. Accumulated fluids in the body, when 
TABLE 2: Herb formulas and ingredients commonly used for reducing side effects of immunosuppressive agents.

\begin{tabular}{|c|c|c|}
\hline Indications & Formulas & Herbs \\
\hline Side effects of steroids & $\begin{array}{l}\text { Six-Ingredient Rehmannia } \\
\text { Decoction (Liu Wei Di } \\
\text { Huang Tang) \& derivatives }\end{array}$ & $\begin{array}{c}\text { Rehmannia glutinosa (Sheng Di Huang), Dioscorea opposita (Shan } \\
\text { Yao), Cornus officinalis (Shan Zhu Yu), Poria cocos (Fu Ling), Alisma } \\
\text { orientale (Ze Xie), Paeonia suffruticosa (Mu Dan Pi), Glehnia littoralis } \\
\text { (Bei Sha Shen), Ophiopogon japonicus (Mai Men Dong), Paeonia } \\
\text { lactiflora (Bai Shao), Lycium barbarum (Gou Qi Zi), Anemarrhena } \\
\text { asphodeloides (Zhi Mu) }\end{array}$ \\
\hline $\begin{array}{l}\text { Side effects of other } \\
\text { immunosuppressants }\end{array}$ & $\begin{array}{l}\text { Bolster the Spleen Decoction } \\
\text { (Shi Pi Yin) Kidney Qi Pill } \\
\text { (Jin Gui Shen Qi Wan) \& } \\
\text { derivatives }\end{array}$ & $\begin{array}{l}\text { Aconitum carmichaelii (Fu Zi), Epimedium (Yin Yang Huo), Morinda } \\
\text { officinalis (Ba Ji Tian), Rehmannia glutinosa (Shu Di Huang), } \\
\text { Atractylodes Macrocephala (Bai Zhu), Codonopsis pilosula (Dang } \\
\text { Shen), Astragalus membranaceus (Huang Qi), Zingiber officinale (Gan } \\
\text { Jiang), Cinnamomum cassia (Gui Zhi), Alpinia Katsumadai (Cao Dou } \\
\text { Kou), Amomum tsao-ko (Cao Guo), Cuscuta australis (Tu Si Zi), } \\
\text { Cistanche deserticola (Rou Cong Rong) }\end{array}$ \\
\hline $\begin{array}{l}\text { Hypoimmunity and } \\
\text { frequent infections }\end{array}$ & $\begin{array}{l}\text { Eight-Treasure Decoction } \\
\text { (Ba Zhen Tang) Jade } \\
\text { Windscreen Powder (Yu } \\
\text { Ping Feng San) }\end{array}$ & $\begin{array}{c}\text { Astragalus membranaceus (Huang Qi), Panax ginseng (Ren Shen), } \\
\text { Atractylodes Macrocephala (Bai Zhu), Glycyrrhiza uralensis (Gan } \\
\text { Cao), Angelica sinensis (Dang Gui), Cordycepssinensis (Dong Chong } \\
\text { Xia Cao), Ganoderma lucidum (Ling Zhi) }\end{array}$ \\
\hline
\end{tabular}

heated by such Yang agents, inevitably generate Damp Heat leading to Yin Deficiency and Yang Excess. This in turn increases the risk of diabetes, hypertension, coronary heart disease, and osteoporosis, with symptoms such as facial flushing, obesity, acne, insomnia, polydipsia, polyphagia, hyperhidrosis, nocturnal emission, and premature ejaculation. On the other hand, immunosuppressive agents such as cyclophosphamide, mycophenolate mofetil, cyclosporin, tacrolimus, and rituximab are Yin agents with cold nature. Long-term use of these drugs damages Yang Qi and further aggravates Yang Deficiency and Yin Excess, manifested as poor appetite, abdominal bloating, nausea and vomiting, shortness of breath and fatigue, cold intolerance, frequent infection, bone marrow suppression, and reproductive suppression, and so on. Severe Yin-Yang imbalance is therefore the core problem facing patients with RNS, contributing to the chronic recurrent pattern of remissions and relapses as well as serious adverse events.

Based on this notion, the TCM strategy for difficultto-treat RNS patients who are experiencing serious toxic effects is to restore the Yin-Yang balance and minimize the doses of immunosuppressive drugs in order to reduce adverse effects, improve therapeutic efficacy, and stabilize the internal environment. For patients receiving steroids, herbal formulas that nourish Yin and suppress Yang are used, with SixIngredient Rehmannia Decoction (Liu Wei Di Huang Tang) as the representative formula in this category. For patients receiving other immunosuppressive agents, herbal formulas that nourish Yang and boost Qi are used, with Bolster the Spleen Decoction (Shi Pi Yin) as the representative formula. For patients with low immunity and frequent infections, herbal formulas that support Upright Qi are used, represented by Eight-Treasure Decoction (Ba Zhen Tang) and Jade Windscreen Powder (Yu Ping Feng San). Depending on the patient's condition, these formulas can be combined and modified to best serve each patient in different phases of disease progression. The commonly used formulas and herb ingredients are listed in Table 2.
Tripterygium wilfordii Hook F (TWHF), a traditional Chinese herb with potent anti-inflammatory and immunosuppressive properties, has been used since the 1980s to treat nephrotic syndrome [42]. Several prospective RCTs reported the effectiveness of Tripterygium glycosides (TG), a fatsoluble extract from the root of TWHF, in supporting the use of steroids for IMN treatment [43-45]. When TG monotherapy and TG-steroid combination therapy were evaluated in 84 patients with IMN, following 12 months of treatment, TG alone improved proteinuria, but the combination therapy achieved much higher remission rate than TG alone $(76.7 \%$ versus $43.9 \%$ ) [43]. Two subsequent studies compared the efficacy of TG-steroid combination and tacrolimus-steroid combination in IMN [44, 45]. Zuo et al. [44] reported that the two treatments achieved comparable effective rate (76.9\% versus $79.9 \%$ ) at 12 months, but TG-steroid group had lower relapse rate than tacrolimus-steroid group $(30.6 \%$ versus 52.5\%) 6 months after discontinuation of treatment. TG-steroid group also had lower serum creatinine doubling rate, indicating less deterioration of renal functions. A recent meta-analysis of 18 studies analyzing 1,236 adult patients with primary nephrotic syndrome demonstrated greater efficacy of TG combined with steroid than steroid monotherapy [46]. Two earlier meta-analysis reviews also showed beneficial effects of TWHF in inducing remission of nephrotic syndrome and RNS $[47,48]$.

The chemical constituents of TWHF and its extracts are complex. Among multiple constituents isolated from TWHF, triptolide is a main active ingredient with important anti-inflammatory and immunosuppressive properties [49]. Triptolide can exert anti-inflammatory functions by reducing the release of many proinflammatory cytokines and mediators such as TNF $\alpha$, IL-6, IL-8, and PGE2 [49]. Suppression of T-lymphocyte functions by triptolide is deemed largely responsible for its immunosuppressive properties, ranging from inducing $\mathrm{T}$-cell apoptosis, inhibiting lymphocyte proliferation, regulating $\mathrm{CD}^{+}$and $\mathrm{CD}^{+}$cells, and reducing IL-2 and interferon- $\gamma$ production [49-53]. 
Its immunosuppressive effects have also been attributed to inhibition of dendritic cell maturation and trafficking [54]. In an experimental model of membranous nephropathy, triptolide significantly reduced proteinuria, protected podocytes from C5b-9-mediated injury, and decreased the expression of desmin, a marker of podocyte injury [55].

Great efforts have been dedicated to exploring the potential of integrating Chinese and Western medicine in improving RNS treatment. From 2001 to 2010, more than 150 RCT studies were published that combined Chinese and Western medicine for RNS treatment, most of which were conducted in China and published in Chinese language journals. A meta-analysis selected 11 of the highest quality trials to evaluate the therapeutic effects of combined Chinese herbal medicine and immunosuppressive therapy in the treatment of RNS [56]. The results indicated that patients receiving herbal medicine in combination with immunosuppressive agents had significantly higher complete or partial remission rates and fewer serious adverse events than those receiving immunosuppressive agents alone.

It is important to point out that despite large numbers of TCM clinical trials in this field, high-quality RCT data are limited. Most studies so far were not double-blinded and had small sample sizes (fewer than 100 participants). Many lack details of cointervention and randomization methods. Overall there is a paucity of strong clinical evidence by the standards of modern medicine. In fact, how to design and conduct RCTs to evaluate the efficacy and safety of TCM in accordance with evidence-based medicine is a challenging task facing TCM researchers worldwide (a comprehensive discussion and review of this topic was undertaken by Fung and Linn [57]). Nevertheless, the large body of clinical data and real-world experience available to date has supported the benefits of integrating TCM and conventional medicine for the treatment of RNS.

\section{Promising Herb-Drug Combinations for RNS}

Extensive research in Chinese herbal medicine has identified many active ingredients that have immunosuppressive activity or can improve existing immunosuppressive therapies for treating autoimmune kidney diseases. Of special note are two herbal ingredients and the herb-drug combinations that have been investigated extensively in preclinical and clinical studies. Convincing evidence indicates that they can improve the therapeutic effects and reduce adverse effects of immunosuppressive agents. These two combinations are Wuzhi capsule with tacrolimus and tetrandrine with glucocorticoid.

5.1. Wuzhi-Tacrolimus Combination. Of calcineurin inhibitors, tacrolimus is preferred over cyclosporine for treating many types of autoimmune diseases and for preventing graft rejection after organ transplantation. This is largely owing to the fact that tacrolimus has consistently demonstrated better therapeutic effects and fewer adverse effects than cyclosporin in most comparative studies [58-62]. However, narrow therapeutic index and unpredictable bioavailability in humans (5\%-67\% with average of $27 \%$ ) have made tacrolimus administration challenging for clinical practice [63]. The high cost of tacrolimus also limits its use.

Wuzhi capsule is an ethanol extract preparation of Schisandra sphenanthera (Nan-Wuweizi), a Chinese herb traditionally used for treating hepatitis, liver/kidney deficiency, and neurasthenia. The main active ingredients are schisandrin, schizandrol B, schisantherin A, schisanhenol, and deoxyschizandrin. Owing to its liver-protective, detoxifying, antioxidant, and antitumor activities [64], Wuzhi has been approved for coadministration with tacrolimus for the treatment of drug-induced hepatitis in organ transplant recipients in China $[65,66]$.

Clinical observations first recognized higher blood levels of tacrolimus when used in combination with Wuzhi. Further studies in humans [67] and animals [68] have confirmed this finding. A meta-analysis [67] evaluating the effects of Wuzhi on tacrolimus pharmacokinetics examined ten RCTs published between 2004 and 2014 that involved 491 patients including mostly kidney or liver transplant recipients and a small number of healthy subjects. Compared with tacrolimus alone, the Tacrolimus-Wuzhi combination significantly increased the plasma concentration of tacrolimus and decreased the dosage of tacrolimus required to maintain the desirable blood concentrations following intervention for one month and three months. These results indicate that Wuzhi capsule can increase the plasma concentration and bioavailability of tacrolimus. The effect appears to be mediated by inhibiting two pathways involved in tacrolimus metabolism, CYP3A and P-glycoprotein, expressed on the intestinal epithelial cells $[69,70]$.

Based on its successful use in organ transplantation, the use of Tacrolimus-Wuzhi combination was explored for the treatment of autoimmune kidney diseases. In a randomized trial of RNS due to IMN, 60 patients were treated for more than six months with tacrolimus-corticosteroids or tacrolimus-corticosteroids-Wuzhi [71]. The two groups showed similar remission rates, while the group containing Wuzhi used lower doses of tacrolimus and therefore had a higher cost-effectiveness. A recent single-center retrospective study compared tacrolimus alone with the Tacrolimus-Wuzhi combination in 60 patients with autoimmune glomerular disease [72]. The results demonstrated that Wuzhi capsule not only significantly reduced the dosage of tacrolimus required to maintain an effective blood concentration, but also resulted in a higher remission rate $(86.7 \%$ versus $70.0 \%)$ and shorter time to achieve partial remission (2.60 months versus 5.22 months).

All studies to date $[65-67,71,72]$ showed that Wuzhi does not increase adverse effects of tacrolimus. In fact, some studies reported improvement in liver functions when Wuzhi was added [67, 72], a finding that is consistent with its known liver-protective activity. Therefore, Wuzhi capsule is a promising tacrolimus-sparing agent for RNS treatment to improve tacrolimus bioavailability, clinical outcome, and pharmacoeconomics.

5.2. Tetrandrine-Glucocorticoid Combination. Tetrandrine is another herb ingredient that has received considerable 
attention with increasing numbers of studies dedicated to understanding its therapeutic effects and biological activities [73]. Tetrandrine is the main active ingredient of Stephania tetrandra S. Moore, a key herb known for its effectiveness in reducing edema. Historically it has been used in the treatment of a wide range of inflammatory and autoimmune diseases. Since 1981 it has been approved in China for treating silicosis and rheumatoid arthritis [74]. The purported immunomodulatory activity of tetrandrine has presumably qualified it as a candidate and as an alternative diseasemodifying antirheumatic drug (DMARD). Synergistic effects have been observed between tetrandrine and DMARDs such as tacrolimus and cyclosporin in patients with rheumatoid arthritis [75].

Despite abundant clinical evidence showing the benefit of tetrandrine in supporting immunosuppressive treatment of autoimmune diseases, mechanistic studies have been largely lacking. Of note is one earlier report by Seow et al. that demonstrated in vitro suppression of mitogen-induced lymphoproliferative responses and antibody production by tetrandrine [76].

Based on our clinical success using herbal formulas containing Stephania tetrandra in the treatment of edema and chronic kidney disease [35], we proposed that tetrandrine possesses direct immunosuppressive activity or can potentiate the effect of corticosteroids. We therefore carried out studies to investigate the potential synergistic interaction between tetrandrine and methylprednisolone and the possible mechanisms of action.

Mitogen-activated human peripheral blood mononuclear cells (PBMCs) are preferred over isolated T cells as an in vitro model for studying the human immune network. We first tested the immunosuppressive effect of methylprednisolone combined with tetrandrine in PBMCs from healthy human subjects [77]. Tetrandrine significantly decreased the half maximal inhibitory concentration $\left(\mathrm{IC}_{50}\right)$ value of methylprednisolone even at the lowest concentration of $0.3 \mathrm{nM}$, and no toxic effect was observed with tetrandrine even at the high concentration of $300 \mathrm{nM}$. Tetrandrine and methylprednisolone both suppressed the production of proinflammatory cytokines TNF- $\alpha$ and IL-6, and the combination showed stronger inhibitory effect. Tetrandrine appears to potentiate the immunosuppressive activity of methylprednisolone via at least two mechanisms. First, it inhibits the function of drug efflux pump P-glycoprotein 170 in T cells and thus increased the intracellular methylprednisolone concentration. Second, tetrandrine in combination with methylprednisolone synergistically inhibits the phosphorylation of mitogen-activated protein kinase family, specifically ERK1/2. However, $\mathrm{CD}^{+}$ $\mathrm{CD} 25^{+}$Foxp $^{+}$regulatory $\mathrm{T}$ cells targeted by some other immunosuppressive drugs such as methotrexate are not affected by tetrandrine and methylprednisolone.

Subsequently, we evaluated the immunosuppressive pharmacodynamics of tetrandrine alone and in combination with methylprednisolone in PBMCs of hemodialysis patients [78]. Tetrandrine alone inhibited the proliferation of PBMCs, with a median (range) $\mathrm{IC}_{50}$ value of $1.61(1.04-4.79) \mu \mathrm{M}$. At lower concentrations $(0.3-300 \mathrm{nM})$, tetrandrine significantly decreased the $\mathrm{IC}_{50}$ value of methylprednisolone. According to a study on the pharmacokinetics of tetrandrine, the maximum blood concentration of tetrandrine after ingesting $40 \mathrm{mg}$ tetrandrine could reach $17 \mu \mathrm{M}$ in healthy volunteers [79]. Thus tetrandrine can augment the immunosuppressive effect of methylprednisolone at clinically relevant doses without risks of side effects.

Collectively, our results demonstrate that tetrandrine is not only an immune inhibitor by itself, but also synergistically potentiates the immunosuppressive efficacy of glucocorticoids in healthy subjects and hemodialysis patients. This finding provides a mechanistic explanation to the observed clinical benefits of Stephania tetrandra in autoimmune diseases. It also offers a clear rationale for using the combination of tetrandrine and glucocorticoids to reduce steroid resistance and attenuate toxic side effects of steroids.

\section{Limitations and Future Studies}

It is worth noting that some Chinese herbs mentioned above are associated with potential side effects. Stephania tetrandra S. Moore (Fang Ji) is a safe herb. However, historically Aristolochia fangchi (Guang Fang Ji) was sometimes mistakenly used as Stephania tetrandra S. Moore, partly due to their similar Chinese names. This contributed to aristolochic acid nephropathy events first discovered in Belgium in 1993 [80] and later found widely in China and other Asian regions [81]. Although most countries have banned Aristolochia fangchi, we should remain cautious because herbs containing aristolochic acid are still used in traditional herbal remedies [82]. Tripterygium wilfordii Hook F. and its extract triptolide, as potent immunosuppressive agents, share features common to immunosuppressants including toxicity. Shortterm administration of the low doses traditionally used in TCM practice does not appear to have toxic effects. However, long-term high-dose treatments can cause liver dysfunction, leukopenia, and damage of the reproductive system [83]. Moreover, TCM emphasizes that herb medicine be prescribed based on the comprehensive differential diagnosis of a patient's conditions guided by TCM theories. Otherwise, if herbs are used inappropriately, even nontoxic herbs can cause adverse effects. Therefore, to avoid and minimize side effects, Chinese herbal prescription should follow the diagnosis and treatment principles of TCM.

Current research in this field is limited in several aspects. First, from the perspective of evidence-based medicine, many clinical trials have been poorly designed and have yet to provide robust evidence of efficacy. Therefore, more highquality clinical studies with large sample sizes are needed to validate the efficacy and safety of Chinese medicine for RNS treatment. A prevailing methodologic drawback in most clinical trials in TCM has been the exclusion of TCM principles in the study design [57]. When Chinese herbal medicine is used to treat a Western medical diagnosis without following TCM principles, any lack of efficacy can arguably result from the mismatch of treatments and diseases in the TCM framework. Therefore, study designs incorporating TCM theories are necessary to improve future RCTs in TCM. Second, there is a lack of understanding of the molecular mechanisms for herbs that have shown promising results 
in treating RNS. This applies to multiherb formulations, for example, Stephania and Astragalus Decoction, and purified components from single herbs, for example, tetrandrine from Stephania tetrandra S. Moore. Not only is detailed and indepth basic research important to elucidate the mechanisms underlying the therapeutic effects of herbal medicine, but it also has the potential to reveal novel signaling pathways and treatment targets for RNS. Finally, investigations of empirical herbal prescriptions that have been well-tested in clinical practice will likely lead to identification of effective herbs and active compounds not previously recognized.

\section{Summary}

Recent advances in understanding the pathogenesis of RNS have led to development and testing of new immunosuppressive agents. Although this has resulted in higher remission rates in some RNS patients, poor clinical outcomes persist due to its chronic relapsing nature as well as unsatisfactory efficacy and toxic adverse effects of available immunosuppressive regimens. The urgent need for alternative treatments has prompted renewed interests in exploring traditional Chinese medicine for safer and more effective therapies for RNS. These efforts have provided evidence supporting the integration of Chinese herbal medicine and conventional therapies. Chinese herbal medicine can improve clinical symptoms, reduce proteinuria, and protect kidney functions of RNS patients through regulation of the internal environment. Some herbs can modulate immune function by affecting immune cells directly and thus can be used as alternative treatments when existing therapies have failed. Other herbs can be coadministered with steroids and other immunosuppressants and have synergistic effects in improving therapeutic efficacy and decreasing adverse events of existing therapies. Current research in this field has been limited by relatively low quality of clinical trials and lack of mechanistic studies. In the era of evidence-based precision medicine, more high-quality clinical studies with large sample sizes and incorporation of TCM principles in the study design are necessary to validate the efficacy and safety of Chinese medicine. More basic research is needed to elucidate the mechanisms of action underlying the therapeutic effects of herbal medicine for treating RNS. The great potential that traditional Chinese medicine holds for RNS warrants such efforts.

\section{Conflicts of Interest}

The authors have declared that they have no conflicts of interest.

\section{Acknowledgments}

This research was supported by the National Natural Science Foundation of China (Project no. 81573907) and the Research Project for Practice Development of National TCM Clinical Research Bases of China (Project no. JDZX2015194). The authors thank Professor Parker B. Antin for critical reading of the manuscript and constructive comments.

\section{References}

[1] A. A. Eddy and J. M. Symons, "Nephrotic syndrome in childhood," The Lancet, vol. 362, no. 9384, pp. 629-639, 2003.

[2] C. Kodner, "Diagnosis and management of nephrotic syndrome in adults," American Family Physician, vol. 93, no. 6, pp. 479485, 2016.

[3] J. Sedor, M. Kretzler, and D. Taylor-Moon, The Nephrotic Syndrome Study Network: A Rare Disease Network for Precision Medicine in Nephrotic Syndrome, 2014, http://www .checkorphan.org/clinical-trials/detail?nctid=NCT0120900.

[4] V. Tesar, T. Zima, and M. Kalousová, "Pathobiochemistry of nephrotic syndrome," Advances in Clinical Chemistry, vol. 37, pp. 173-218, 2003.

[5] P. Ronco and H. Debiec, "Pathogenesis of membranous nephropathy: Recent advances and future challenges," Nature Reviews Nephrology, vol. 8, no. 4, pp. 203-213, 2012.

[6] A. Kuroki, "M-Type Phospholipase A2 Receptor as Target Antigen in Idiopathic Membranous Nephropathy NEJM," New England Journal of Medicine, vol. 361, no. 1, pp. 11-21, 2009.

[7] N. M. Tomas, B. L. C. Meyer-Schwesinger Jr. et al., "Thrombospondin type-1 domain-containing 7A in idiopathic membranous nephropathy," New England Journal of Medicine, vol. 372, no. 11, Article ID 2287, 2014.

[8] R. J. Maas, J. K. Deegens, B. Smeets, M. J. Moeller, and J. F. Wetzels, "Minimal change disease and idiopathic FSGS: Manifestations of the same disease," Nature Reviews Nephrology, vol. 12, no. 12, pp. 768-776, 2016.

[9] A. B. Fogo, "Causes and pathogenesis of focal segmental glomerulosclerosis," Nature Reviews Nephrology, vol. 11, no. 2, pp. 7687, 2015.

[10] Kidney Disease: Improving Global Outcomes (KDIGO) Glomerulonephritis Work Group, "KDIGO clinical practice guideline for glomerulonephritis," Kidney International Supplements, vol. 2, no. 2, pp. 139-274, 2012.

[11] Z. Zhao, G. Liao, Y. Li, S. Zhou, and H. Zou, "The efficacy and safety of rituximab in treating childhood refractory nephrotic syndrome: A meta-analysis," Scientific Reports, vol. 5, article no. 8219, 2015.

[12] R. K. Chandra, "Refractory nephrotic syndrome," Nihon Rinsho Japanese Journal of Clinical Medicine, vol. 62, no. 10, pp. 17941799, 2004.

[13] K. L. Gibson, P. Hansrivijit, and M. E. Ferris, "Emerging Agents for the Management of Nephrotic Syndrome: Progress to Date," Pediatric Drugs, vol. 18, no. 1, pp. 25-29, 2016.

[14] M. Vivarelli, L. Massella, B. Ruggiero, and F. Emma, "Minimal change disease," Clinical Journal of the American Society of Nephrology, vol. 12, no. 2, pp. 332-345, 2017.

[15] L. A. Greenbaum, R. Benndorf, and W. E. Smoyer, "Childhood nephrotic syndrome-current and future therapies," Nature Reviews Nephrology, vol. 8, no. 8, pp. 445-458, 2012.

[16] V. D. B. Ja, P. R. van Dijk, J. M. Hofstra, and J. F. Wetzels, "Longterm outcomes in idiopathic membranous nephropathy using a restrictive treatment strategy," Journal of the American Society of Nephrology, vol. 25, no. 1, pp. 150-158, 2014.

[17] P. Ruggenenti, F. C. Fervenza, and G. Remuzzi, "Treatment of membranous nephropathy: time for a paradigm shift," Nature Reviews Nephrology, vol. 13, no. 9, pp. 563-579, 2017.

[18] G. Remuzzi, C. Chiurchiu, M. Abbate, V. Brusegan, M. Bontempelli, and P. Ruggenenti, "Rituximab for idiopathic membranous nephropathy," The Lancet, vol. 360, no. 9337, pp. 923-924, 2002. 
[19] A. Segarra, M. Praga, N. Ramos et al., "Successful treatment of membranous glomerulonephritis with rituximab in calcineurin inhibitor-dependent patients," Clinical Journal of the American Society of Nephrology, vol. 4, no. 6, pp. 1083-1088, 2009.

[20] K. Iijima, D. M. Sako, K. Nozu et al., "Rituximab for childhoodonset, complicated, frequently relapsing nephrotic syndrome or steroid-dependent nephrotic syndrome: a multicentre, doubleblind, randomised, placebo-controlled trial," The Lancet, vol. 384, no. 9950, pp. 1273-1281, 2014.

[21] P. Ruggenenti, B. Ruggiero, P. Cravedi et al., "Rituximab in steroid-dependent or frequently relapsing idiopathic nephrotic syndrome," Journal of the American Society of Nephrology, vol. 25, no. 4, pp. 850-863, 2014.

[22] C. D. Garcia, V. B. Bittencourt, A. Tumelero, J. S. Antonello, D. Malheiros, and V. D. Garcia, "Plasmapheresis for Recurrent Posttransplant Focal Segmental Glomerulosclerosis," Transplantation Proceedings, vol. 38, no. 6, pp. 1904-1905, 2006.

[23] T. P. Andolino and J. Reid-Adam, "Nephrotic syndrome," Pediatrics in Review, vol. 36, no. 3, pp. 117-126, 2015.

[24] S. Yin, T. He, Y. Li et al., "Rituximab shows no effect on remission in patients with refractory nephrotic syndrome: A MOOSE-compliant meta-analysis," Medicine (United States), vol. 95, no. 50, p. e5320, 2016.

[25] F. Iorember, D. Aviles, M. Kallash, and O. Bamgbola, "Cost analysis on the use of rituximab and calcineurin inhibitors in children and adolescents with steroid-dependent nephrotic syndrome," Pediatric Nephrology.

[26] Q. Q. Wang, Selected Readings from the Yellow Emperor's Inner Classic, China Press of Traditional Chinese Medicine, Beijing, China, 2nd edition, 2007.

[27] X. Q. Wang, C. J. Wang, and J. Yuan, "Immune glomerular diseases difficulties and Chinese medicine treatment strategies," Chinese Journal of Integrated Traditional and Western Nephrology, vol. 11, no. 2, pp. 169-171, 2010.

[28] V. T. Devita, T. S. Lawrence, and S. A. Rosenberg, Cancer: principles practice of oncology : primer of the molecular biology of cancer, Wolters Kluwer Health, 2nd edition, 2015.

[29] Z. J. Zhang, Synopsis of prescriptions of the golden chamber (Jinkui yaolue fanglun), Beijing Science and Technology, Beijing, China, 2010.

[30] A. Peng, Y. Gu, and S. Y. Lin, "Herbal treatment for renal diseases," Annals, Academy of Medicine, Singapore, vol. 34, no. 1, pp. 44-51, 2005.

[31] J. C. Meng, "Astragalus is effective medicine for chronic proteinuria," Jiangsu Chinese Medicine Journal, vol. 16, no. 1, pp. 316-316, 1995.

[32] J. F. Shi, H. W. Zhu, C. Zhang, F. Bian, P. J. Shan, and W. $\mathrm{Lu}$, "Therapeutic effect of Astragalus in patients with chronic glomerulonephritis," Acta Univ Medicinalis Secondae Shanghai, vol. 22, no. 3, pp. 245-247, 2002.

[33] M. S. Ahmed, S. H. Hou, M. C. Battaglia, M. M. Picken, and D. J. Leehey, "Treatment of idiopathic membranous nephropathy with the herb Astragalus membranaceus," American Journal of Kidney Diseases, vol. 50, no. 6, pp. 1028-1032, 2007.

[34] D. J. Leehey, T. Casini, and D. Massey, "Remission of Membranous Nephropathy After Therapy With Astragalus Membranaceus," American Journal of Kidney Diseases, vol. 55, no. 4, p. 772, 2010.

[35] Y. J. Wang, L. Q. He, W. Sun, Y. Lu, and X. Q. Wang, "Optimized project of traditional Chinese medicine in treating chronic kidney disease stage 3: a multicenter double-blinded randomized controlled trial," Journal of Ethnopharmacology, vol. 139, no. 3, pp. 757-764, 2012.

[36] X. R. Chen, "Clinical observation on curative effect of primary nephrotic syndrome treated with JiaweiFangjiHuangqi Tang and its effects on serum IL-10 and TNF- $\alpha$," Traditional Chinese Medicine Technology, vol. 23, no. 4, pp. 393-395, 2016.

[37] W. J. Du and A. J. Zhan, "Effect of FangjiHuangqi Tang on serum TNF-a and IL6 in primary nephrotic syndrome," Henan Traditional Chinese Medicine, vol. 34, no. 5, pp. 805-806, 2014.

[38] J. T. Nie, "Efficacy of fangjihuangqi decoction in treatment of patients with primary nephrotic syndrome in edema period and its influence on $24 \mathrm{hr}$ urinary protein, serum albumin and lipid," Jounral of Clinical Medicine in Practice, vol. 21, no. 1, pp. 48-51, 2017.

[39] L. Han, Y. Y. Deng, Y. P. Chen et al., "Clinical therapeutic research of idiopathic membranous nephropathy by YiqiHuoxueHuashi Project and analysis of correlative influencing factors," Chinese Journal Integrated Traditional and Western Nephrolopgy, vol. 11, no. 10, pp. 879-883, 2010.

[40] L. Wang, Y. P. Chen, Y. Y. Deng et al., "The treatment of membranous nephropathy by, YiqiHuoxueHuashi Project," Chinese Journal Integrated Traditional and Western Nephrolopgy, vol. 7, no. 8, pp. 393-396, 2006.

[41] Y. Chen, Y. Deng, Z. Ni et al., "Efficacy and safety of traditional chinese medicine (Shenqi particle) for patients with idiopathic membranous nephropathy: a multicenter randomized controlled clinical trial," American Journal of Kidney Diseases, vol. 62, no. 6, pp. 1068-1076, 2013.

[42] S. Y. Chen, "Effect of Tripterygium wilfordii on the remission of proteinuria in patients with nephrotic syndrome," Chinese Journal of Modern Developments in Traditional Medicine, vol. 5, no. 3, pp. 164-166, 1985.

[43] Z. H. Liu, S. J. Li, Y. Wu et al., "Treatment of membranous nephropathy with Tripterygium wilfordii and steroid: a prospective randomized control trial," Chinese Journal of Nephrology Dialysis Transplantation, vol. 18, no. 4, pp. 303-309, 2009.

[44] K. Zuo, S. J. Li, Y. Wu et al., "Treatment of idiopathic membranous nephropathy with tripterygium wilfordii hook F: a prospective randomized control trial," Chinese Journal of Nephrology Dialysis Transplantation, vol. 23, no. 6, pp. 507-511, 2014.

[45] S. Liu, X. Li, H. Li, Q. Liang, J. Chen, and J. Chen, "Comparison of tripterygium wilfordii multiglycosides and tacrolimus in the treatment of idiopathic membranous nephropathy: A prospective cohort study," BMC Nephrology, vol. 16, no. 1, Article ID 0199-x, 2015.

[46] Y. Y. Zhang, S. F. Zeng, B. Yan, D. Z. Chen, F. N. Liu, and L. H. Yin, "Tripterygium Glycosides Combined with Glucocorticoid for Primary Nephrotic Syndrome in Adults:a Meta-analysis," Journal of Chinese General Practice, vol. 20, no. 14, pp. 1742-1748, 2017.

[47] G. Xu, W. Tu, D. Jiang, and C. Xu, “Tripterygium wilfordii Hook F treatment for idiopathic refractory nephrotic syndrome in adults: a meta-analysis," Nephron Clinical Practice, vol. 111, no. 4, pp. c223-c228, 2009.

[48] Y. Chen, Z. Gong, X. Chen et al., "Tripterygium wilfordii Hook F (a traditional Chinese medicine) for primary nephrotic syndrome.", Cochrane Database of Systematic Reviews, vol. 8, p. CD008568, 2013.

[49] B. J. Chen, “Triptolide, a novel immunosuppressive and antiinflammatory agent purified from a Chinese herb Tripterygium 
Wilfordii Hook F,' Leukemia \& Lymphoma, vol. 42, no. 3, pp. 253-265, 2001.

[50] R. J. Pei, L. H. Qi, and X. J. Liu, "Mechanism of immunosuppressive effects of triptolide," Chinese Pharmacological Bulletin, vol. 9, no. 1, pp. 68-72, 1993.

[51] X. M. Guo, C. Z. Wang, and Zhangjiakou, "Effect of tripholide on peripheral blood lymphocytes IL-5, GM-CSF mRNA and CD4 +, CD8 + expression in allergic guinea pigs," Chinese Journal of Pathophysiology, vol. 15, no. 11, pp. 1003-1005, 1999.

[52] Y. Yang, Z.-H. Liu, E. Tolosa, J.-W. Yang, and L.-S. Li, “Triptolide induces apoptotic death of T lymphocyte," International Journal of immunopharmacology, vol. 40, no. 2, pp. 139-149, 1998.

[53] M. A. Chan, J. E. Kohlmeier, M. Branden, M. Jung, and S. $\mathrm{H}$. Benedict, "Triptolide is more effective in preventing T cell proliferation and interferon-gamma production than is FK506," Phytotherapy Research, vol. 13, no. 6, pp. 464-467, 1999.

[54] X. Chen, T. Murakami, J. J. Oppenheim, and O. M. Z. Howard, "Triptolide, a constituent of immunosuppressive Chinese herbal medicine, is a potent suppressor of dendritic-cell maturation and trafficking," Blood, vol. 106, no. 7, pp. 2409-2416, 2005.

[55] Z.-H. Chen, W.-S. Qin, C.-H. Zeng et al., "Triptolide reduces proteinuria in experimental membranous nephropathy and protects against C5b-9-induced podocyte injury in vitro," Kidney International, vol. 77, no. 11, pp. 974-988, 2010.

[56] P. J. Lv, L. B. Wei, L. Chen et al., "Meta-Analysis of combined Chinese and Western Medicine in Primary Refractory Nephritic Syndrome," Journal of Mathematical Medicine, vol. 25, no. 3, pp. 332-335, 2012.

[57] F. Y. Fung and Y. C. Linn, "Developing traditional Chinese medicine in the era of evidence-based medicine: current evidences and challenges," Evidence-Based Complementary and Alternative Medicine, vol. 2015, Article ID 425037, 9 pages, 2015.

[58] H. Fu, G. Qian, and Z. Jiang, "Comparison of second-line immunosuppressants for childhood refractory nephrotic syndrome: a systematic review and network meta-analysis," Journal of Investigative Medicine, vol. 65, no. 1, pp. 65-71, 2016.

[59] E. Gathogo, M. Harber, S. Bhagani et al., "Impact of tacrolimus compared with cyclosporin on the incidence of acute allograft rejection in human immunodeficiency virus-positive kidney transplant recipients," Transplantation, vol. 100, no. 4, pp. 871878, 2016.

[60] M. Kamel, M. Kadian, T. Srinivas, D. Taber, and M. A. Salas, "Tacrolimus confers lower acute rejection rates and better renal allograft survival compared to cyclosporine," World Journal of Transplantation, vol. 6, no. 4, p. 697, 2016.

[61] S. M. Goring, A. R. Levy, I. Ghement et al., "A network meta-analysis of the efficacy of belatacept, cyclosporine and tacrolimus for immunosuppression therapy in adult renal transplant recipients," Current Medical Research and Opinion, vol. 30, no. 8, pp. 1473-1487, 2014.

[62] M. Rodríguez-Perálvarez, M. Guerrero-Misas, D. Thorburn, B. R. Davidson, E. Tsochatzis, and K. S. Gurusamy, "Maintenance immunosuppression for adults undergoing liver transplantation: A network meta-analysis," Cochrane Database of Systematic Reviews, vol. 3, no. 3, Article ID CD011639, 2017.

[63] S. Tremblay and R. R. Alloway, "Clinical Evaluation of Modified Release and Immediate Release Tacrolimus Formulations," The AAPS Journal, vol. 19, no. 5, pp. 1332-1347, 2017.

[64] H. D. Chen, H. He, and Y. L. Zhang, "Baiying Decoction in the treatment of 43 cases of chronic hepatitis B," Journal of Anhui
Traditional Chinese Medical College, vol. 21, no. 6, pp. 17-19, 2002 (Chinese).

[65] S. P. Xie, Q. Yan, and H. Z. Chen, "Study on the clinical application of wuzhi capsule after renal transplantation," Chinese Journal of Integrated Traditional Western Medicine, vol. 31, no. 9, pp. 1213-1215, 2011.

[66] K. Wang, Q. S. Qu, Y. X. Zhang, S. Z. Miao, and X. Jiang, "Effects of Wuzhi capsule on blood concentration of tacrolimus after renal transplantation," J Biol Regul Homeost Agents, vol. 30, no. 1, pp. 155-159, 2016.

[67] M. Zeng, D. I. Xiao-Hui, W. J. Zhao, Y. G. Yang, and D. O. Pharmacology, "Effect of Wuzhi capsules (or tablets) on blood concentration and pharmacokinetics of tacrolimus in recipients of liver (or kidney) transplantation:a meta-analysis," Pharmaceutical Journal of Chinese Peoples Liberation Army, vol. 30, no. 5, pp. 405-409, 2014.

[68] H. Wei, X. Tao, P. Di et al., "Effects of traditional chinese medicine wuzhi capsule on pharmacokinetics of tacrolimus in rats," Drug Metabolism and Disposition, vol. 41, no. 7, pp. 1398 1403, 2013.

[69] H. Iwata, Y. Tezuka, S. Kadota, A. Hiratsuka, and T. Watabe, "Identification and characterization of potent CYP3A4 inhibitors in Schisandra fruit extract," Drug Metabolism and Disposition, vol. 32, no. 12, pp. 1351-1358, 2004.

[70] W.-F. Fong, C.-K. Wan, C.-Y. Zhu et al., "Schisandrol A from Schisandra chinensis reverses P-glycoprotein-mediated multidrug resistance by affecting Pgp-substrate complexes," Planta Medica, vol. 73, no. 3, pp. 212-220, 2007.

[71] Z. Sun, M. Ren, Q. Wu, and X. Du, "Co-administration of Wuzhi capsules and tacrolimus in patients with idiopathic membranous nephropathy: Clinical efficacy and pharmacoeconomics," International Urology and Nephrology, vol. 46, no. 10, pp. 19771982, 2014.

[72] N. Yang, J. L. Chen, S. F. Liu, and H. Xie, “Tacrolimus alone or combined Wuzhi capsules for the treatment of autoimmune glomerular disease in a single-center retrospective study," Journal of Medicine and Philosophy, vol. 38, no. 10, pp. 34-36, 2017.

[73] N. Bhagya and K. R. Chandrashekar, "Tetrandrine-A molecule of wide bioactivity," Phytochemistry, vol. 125, pp. 5-13, 2016.

[74] Z. L. Gao, "A new drug to cure silicosis-Tetrandrine," Radiation Protection Bulletin, vol. 2, p. 56, 1981.

[75] L.-J. Ho and J.-H. Lai, "Chinese herbs as immunomodulators and potential disease-modifying antirheumatic drugs in autoimmune disorders," Current Drug Metabolism, vol. 5, no. 2, pp. 181-192, 2004.

[76] W. K. Seow, A. Ferrante, D. B. H. Goh, A. H. Chalmers, S.-Y. Li, and Y. H. Thong, "In vitro immunosuppressive properties of the plant alkaloid tetrandrine," International Archives of Allergy and Applied Immunology, vol. 85, no. 4, pp. 410-415, 1988.

[77] W. Xu, K. Meng, Y. Tu et al., "Tetrandrine potentiates the glucocorticoid pharmacodynamics via inhibiting P-glycoprotein and mitogen-activated protein kinase in mitogen-activated human peripheral blood mononuclear cells," European Journal of Pharmacology, vol. 807, pp. 102-108, 2017.

[78] W. C. Xu, K. Meng, J. Kusano et al., "Immunosuppressive efficacy of tetrandrine combined with methylprednisolone against mitogen-activated peripheral blood mononuclear cells of hemodialysis patients," Clinical Experimental Pharmacology Physiology, vol. 44, no. 9, pp. 924-931, 2017.

[79] Q. Dong, "Study on the pharmacokinetics of tetrandrine tablets in healthy volunteers," Journal of Clinical Medicine in Practice, vol. 15, no. 7, pp. 83-85, 2011. 
[80] M. Vanhaelen, R. Vanhaelen-Fastre, P. But, and J. Vanherweghem, "Identification of aristolochic acid in Chinese herbs," The Lancet, vol. 343, article 174, 1994.

[81] H.-Y. Yang, P.-C. Chen, and J.-D. Wang, "Chinese herbs containing aristolochic acid associated with renal failure and urothelial carcinoma: A review from epidemiologic observations to causal inference," BioMed Research International, vol. 2014, Article ID 569325, 2014.

[82] N. Anandagoda and G. M. Lord, "Preventing aristolochic acid nephropathy," Clinical Journal of the American Society of Nephrology, vol. 10, no. 2, pp. 167-168, 2015.

[83] C. Xi, S. Peng, Z. Wu, Q. Zhou, and J. Zhou, "Toxicity of triptolide and the molecular mechanisms involved," Biomedicine \& Pharmacotherapy, vol. 90, pp. 531-541, 2017. 




The Scientific World Journal


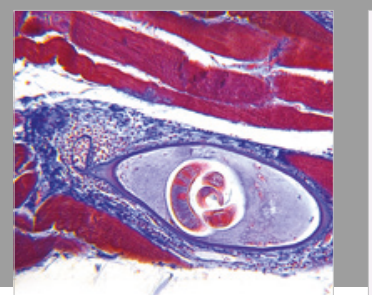

Gastroenterology Research and Practice



\section{Hindawi}

Submit your manuscripts at

www.hindawi.com
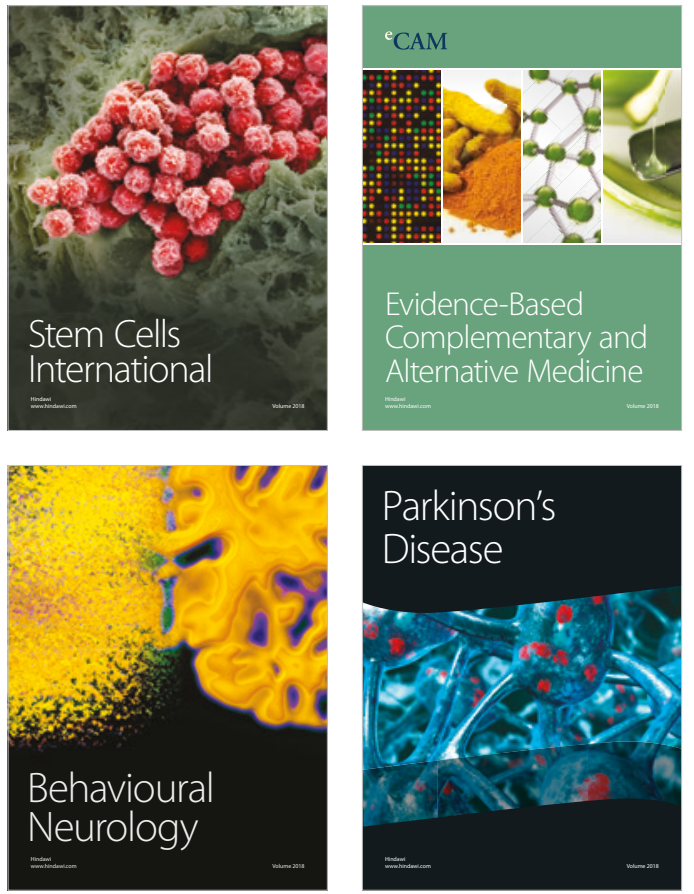

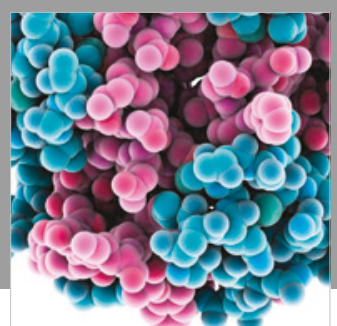

ournal of

Diabetes Research

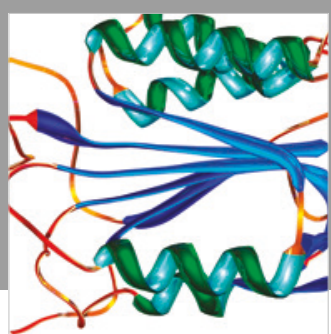

Disease Markers
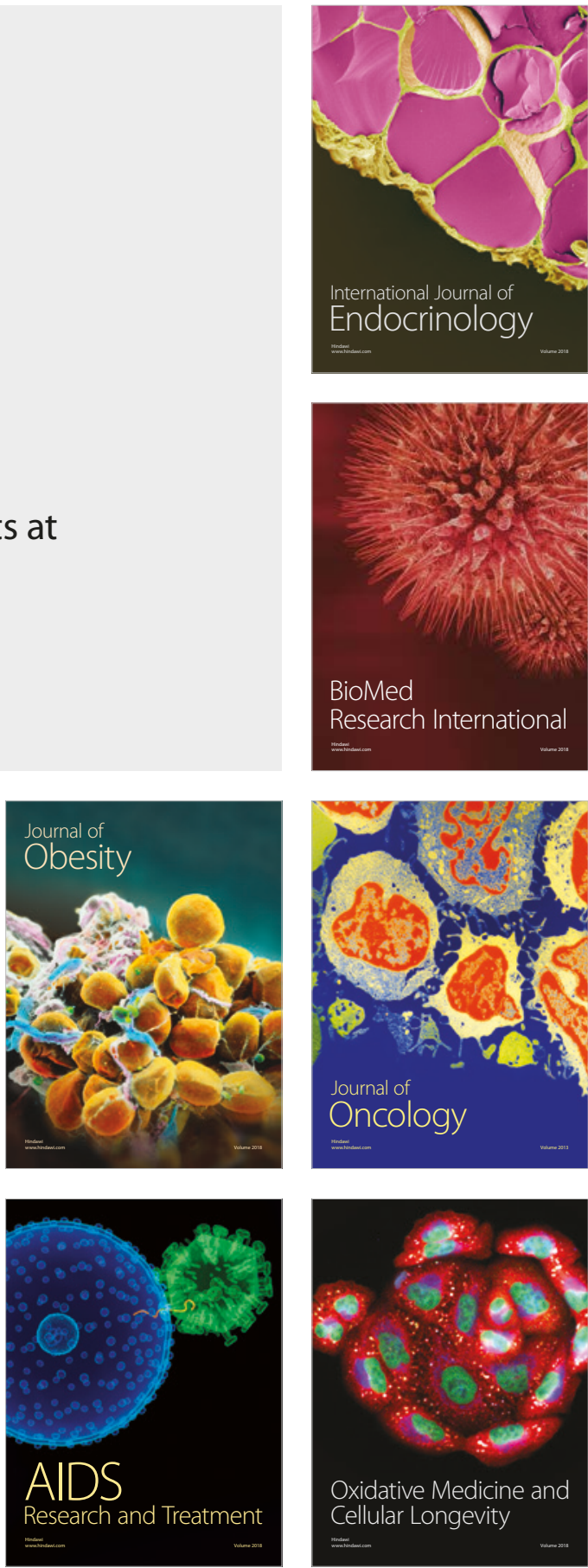